Acta Crystallographica Section E

Structure Reports

Online

ISSN 1600-5368

\section{3-Cyanoanilinium chloride}

\section{Xiao-Chun Wen}

Ordered Matter Science Research Center, College of Chemistry and Chemical Engineering, Southeast University, Nanjing 210096, People's Republic of China

Correspondence e-mail: fudavid88@yahoo.com.cn

Received 30 June 2008; accepted 3 July 2008

Key indicators: single-crystal X-ray study; $T=298 \mathrm{~K}$; mean $\sigma(\mathrm{C}-\mathrm{C})=0.004 \AA$; $R$ factor $=0.053 ; w R$ factor $=0.157 ;$ data-to-parameter ratio $=17.8$.

In the title salt, $\mathrm{C}_{7} \mathrm{H}_{7} \mathrm{~N}_{2}{ }^{+} \cdot \mathrm{Cl}^{-}$, all non- $\mathrm{H}$ atoms of the cation are essentially coplanar (r.m.s. deviation $=0.005 \AA$ ). In the crystal structure, the organic cations and chloride ions are linked to form a two-dimensional network parallel to the (001) plane by $\mathrm{N}-\mathrm{H} \cdots \mathrm{Cl}$ hydrogen bonds.

\section{Related literature}

For the use of amine derivatives in coordination chemistry, see: Manzur et al. (2007); Ismayilov et al. (2007); Austria et al. (2007).

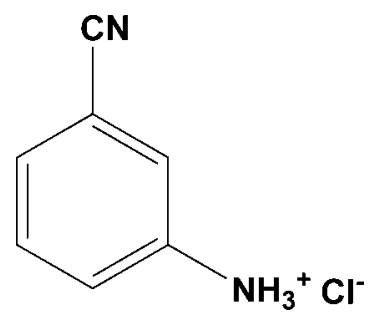

\section{Experimental}

\section{Crystal data}

$\mathrm{C}_{7} \mathrm{H}_{7} \mathrm{~N}_{2}^{+} \cdot \mathrm{Cl}^{-}$

$M_{r}=154.60$

Triclinic, $P \overline{1}$

$a=4.663(3) \AA$ $\gamma=96.22(4)^{\circ}$

$V=368.9(5) \AA^{3}$

$Z=2$

Mo $K \alpha$ radiation

Data collection

Rigaku Mercury2 diffractometer Absorption correction: multi-scan (CrystalClear; Rigaku, 2005)

$T_{\min }=0.901, T_{\max }=0.917$

$\mu=0.43 \mathrm{~mm}^{-1}$

$T=298$ (2) K

$0.25 \times 0.18 \times 0.18 \mathrm{~mm}$

2486 measured reflections 1618 independent reflections 1342 reflections with $I>2 \sigma(I)$ $R_{\text {int }}=0.021$

Refinement

$R\left[F^{2}>2 \sigma\left(F^{2}\right)\right]=0.052$

$w R\left(F^{2}\right)=0.157$

$S=1.07$

1618 reflections

91 parameters

$\mathrm{H}$-atom parameters constrained

$\Delta \rho_{\max }=0.52{\mathrm{e} \AA^{-3}}^{-3}$

$\Delta \rho_{\min }=-0.52{\mathrm{e} \AA^{-3}}^{-3}$

Table 1

Hydrogen-bond geometry $\left(\AA{ }^{\circ}\right)$.

\begin{tabular}{lllll}
\hline$D-\mathrm{H} \cdots A$ & $D-\mathrm{H}$ & $\mathrm{H} \cdots A$ & $D \cdots A$ & $D-\mathrm{H} \cdots A$ \\
\hline $\mathrm{N} 2-\mathrm{H} 9 A \cdots \mathrm{Cl} 1^{\mathrm{i}}$ & 0.89 & 2.61 & $3.111(3)$ & 116 \\
$\mathrm{~N} 2-\mathrm{H} 9 A \cdots \mathrm{Cl}{ }^{\text {ii }}$ & 0.89 & 2.65 & $3.178(3)$ & 119 \\
$\mathrm{~N} 2-\mathrm{H} 9 C \cdots \mathrm{Cl} 1^{\text {iii }}$ & 0.89 & 2.73 & $3.278(3)$ & 121 \\
$\mathrm{~N} 2-\mathrm{H} 9 B \cdots \mathrm{Cl} 1$ & 0.89 & 2.76 & $3.338(3)$ & 124 \\
\hline
\end{tabular}

Symmetry codes: (i) $-x,-y+1,-z$; (ii) $x, y+1, z$; (iii) $-x+1,-y+1,-z$.

Data collection: CrystalClear (Rigaku, 2005); cell refinement: CrystalClear; data reduction: CrystalClear; program(s) used to solve structure: SHELXS97 (Sheldrick, 2008); program(s) used to refine structure: SHELXL97 (Sheldrick, 2008); molecular graphics: SHELXTL (Sheldrick, 2008); software used to prepare material for publication: SHELXTL.

This work was supported by a Start-up Grant from Southeast University to Professor Ren-Gen Xiong.

Supplementary data and figures for this paper are available from the IUCr electronic archives (Reference: CI2624).

\section{References}

Austria, C., Zhang, J. \& Valle, H. (2007). Inorg. Chem. 46, 6283-6290.

Ismayilov, R. H., Wang, W. Z. \& Lee, G. H. (2007). Dalton Trans. pp. 28982907.

Manzur, J., Vega, A. \& Garcia, A. M. (2007). Eur. J. Inorg. Chem. 35, 55005510.

Rigaku (2005). CrystalClear. Rigaku Corporation, Tokyo, Japan.

Sheldrick, G. M. (2008). Acta Cryst. A64, 112-122. 


\section{supporting information}

Acta Cryst. (2008). E64, o1462 [doi:10.1107/S1600536808020485]

\section{3-Cyanoanilinium chloride}

\section{Xiao-Chun Wen}

\section{S1. Comment}

In the past five years, we have focused on the chemistry of amine derivatives because of their multiple coordination modes as ligands to metal ions and for the construction of novel metal-organic frameworks (Manzur et al. 2007; Ismayilov et al. 2007; Austria et al. 2007). We report here the crystal structure of the title compound, 3-cyanobenzenaminium monochloride.

In the title compound (Fig.1), the N2 atom of the amine group is protonated. The nitrile group is coplanar with the benzene ring. Bond lengths and angles lie within normal ranges.

In the crystal structure the organic cation and $\mathrm{Cl}^{-}$ions are linked to form a two-dimensional network parallel to the $(00$ 1) plane (Fig.2) by $\mathrm{N}-\mathrm{H} \cdots \mathrm{Cl}$ hydrogen bonds (Table 1).

\section{S2. Experimental}

3-Cyanobenzenaminium monochloride $(3 \mathrm{mmol})$ was dissolved in ethanol $(20 \mathrm{ml})$. The solution was allowed to evaporate to obtain colourless block-shaped crystals of the title compound.

\section{S3. Refinement}

All $\mathrm{H}$ atoms were fixed geometrically $[\mathrm{C}-\mathrm{H}=0.93 \AA$ and $\mathrm{N}-\mathrm{H}=0.89 \AA]$ and treated as riding, with $U_{\text {iso }}(\mathrm{H})=1.2 \mathrm{U}_{\text {eq }}(\mathrm{C})$ and $1.5 \mathrm{U}_{\mathrm{eq}}(\mathrm{N})$. 


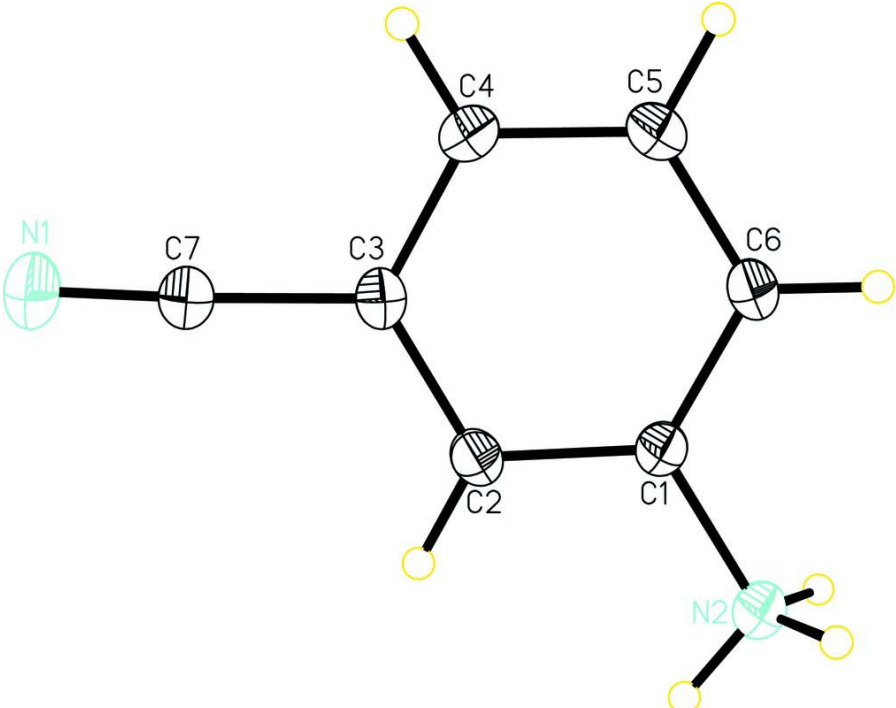

\section{Figure 1}

A view of the title compound with the atomic numbering scheme. Displacement ellipsoids are drawn at the $30 \%$ probability level. 


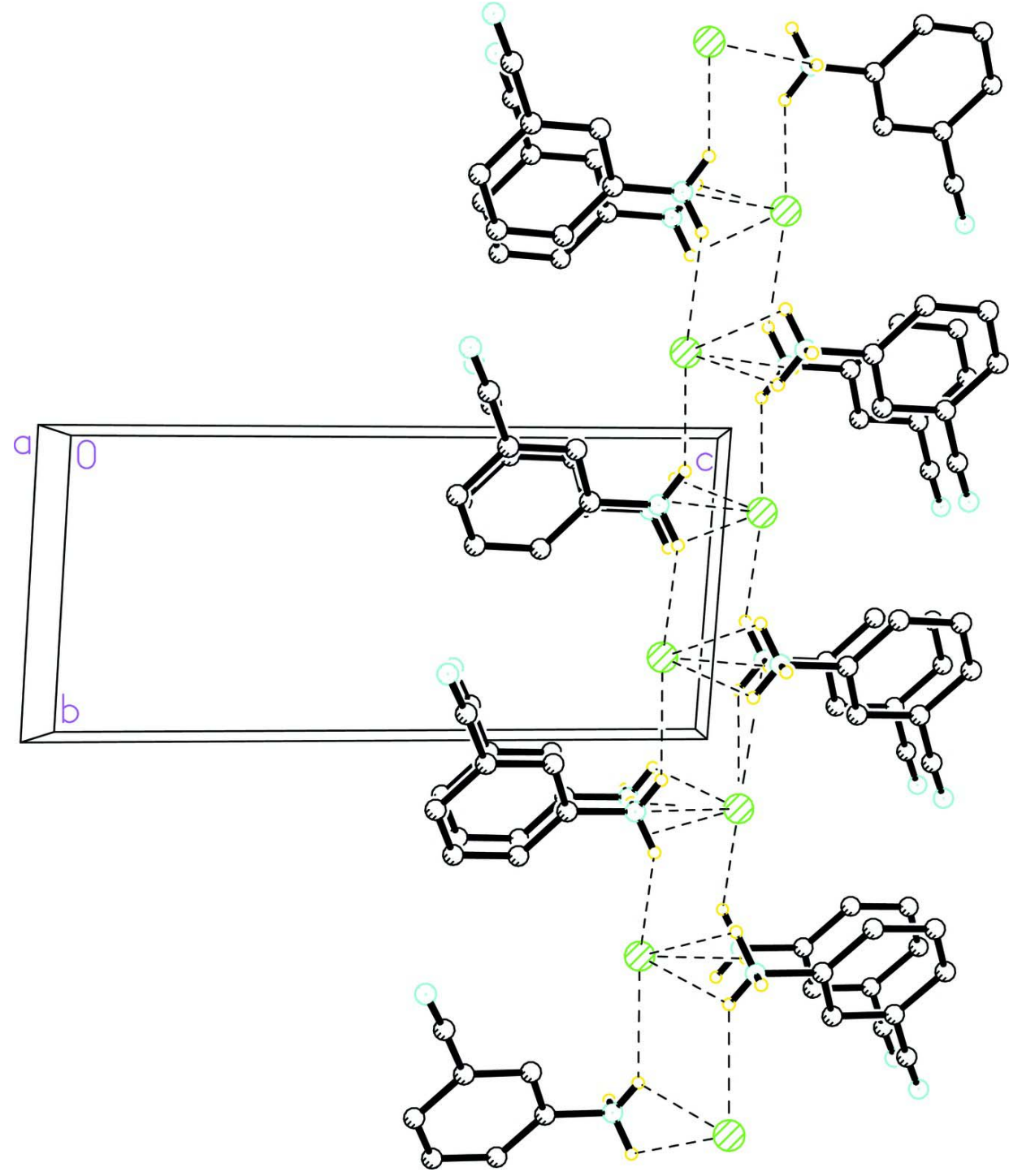

Figure 2

Part of the crystal packing of the title compound viewed along the $a$ axis. $\mathrm{H}$ atoms not involved in hydrogen bonding (dashed lines) have been omitted for clarity.

\section{3-Cyanoanilinium chloride}

Crystal data

$\mathrm{C}_{7} \mathrm{H}_{7} \mathrm{~N}_{2}{ }^{+} \cdot \mathrm{Cl}^{-}$

$M_{r}=154.60$

Triclinic, $P \overline{1}$

Hall symbol: -P 1

$a=4.663$ (3) $\AA$

$b=6.074(5) \AA$

$c=13.212(9) \AA$

$\alpha=93.37(5)^{\circ}$

$\beta=96.201(19)^{\circ}$

$\gamma=96.22(4)^{\circ}$

$V=368.9(5) \AA^{3}$
$Z=2$

$F(000)=160$

$D_{\mathrm{x}}=1.392 \mathrm{Mg} \mathrm{m}^{-3}$

Mo $K \alpha$ radiation, $\lambda=0.71073 \AA$

Cell parameters from 1678 reflections

$\theta=2.3-24.4^{\circ}$

$\mu=0.44 \mathrm{~mm}^{-1}$

$T=298 \mathrm{~K}$

Block, colourless

$0.25 \times 0.18 \times 0.18 \mathrm{~mm}$ 


\section{Data collection}

Rigaku Mercury2 diffractometer

Radiation source: fine-focus sealed tube Graphite monochromator

Detector resolution: 13.6612 pixels $\mathrm{mm}^{-1}$ $\omega$ scans

Absorption correction: multi-scan

(CrystalClear; Rigaku, 2005)

$T_{\min }=0.901, T_{\max }=0.917$

Refinement

Refinement on $F^{2}$

Least-squares matrix: full

$R\left[F^{2}>2 \sigma\left(F^{2}\right)\right]=0.052$

$w R\left(F^{2}\right)=0.157$

$S=1.07$

1618 reflections

91 parameters

0 restraints

Primary atom site location: structure-invariant direct methods
2486 measured reflections

1618 independent reflections

1342 reflections with $I>2 \sigma(I)$

$R_{\text {int }}=0.021$

$\theta_{\max }=27.5^{\circ}, \theta_{\min }=3.1^{\circ}$

$h=-6 \rightarrow 6$

$k=-7 \rightarrow 5$

$l=-16 \rightarrow 17$

\section{Special details}

Geometry. All e.s.d.'s (except the e.s.d. in the dihedral angle between two 1.s. planes) are estimated using the full covariance matrix. The cell e.s.d.'s are taken into account individually in the estimation of e.s.d.'s in distances, angles and torsion angles; correlations between e.s.d.'s in cell parameters are only used when they are defined by crystal symmetry. An approximate (isotropic) treatment of cell e.s.d.'s is used for estimating e.s.d.'s involving 1.s. planes.

Refinement. Refinement of $F^{2}$ against ALL reflections. The weighted $R$-factor $w R$ and goodness of fit $S$ are based on $F^{2}$, conventional $R$-factors $R$ are based on $F$, with $F$ set to zero for negative $F^{2}$. The threshold expression of $F^{2}>\sigma\left(F^{2}\right)$ is used only for calculating $R$-factors(gt) etc. and is not relevant to the choice of reflections for refinement. $R$-factors based on $F^{2}$ are statistically about twice as large as those based on $F$, and $R$ - factors based on ALL data will be even larger.

Fractional atomic coordinates and isotropic or equivalent isotropic displacement parameters $\left(\hat{A}^{2}\right)$

\begin{tabular}{lllll}
\hline & $x$ & $y$ & $z$ & $U_{\text {iso }} * U_{\mathrm{eq}}$ \\
\hline C11 & $0.38158(16)$ & $0.25924(11)$ & $0.07001(5)$ & $0.0414(3)$ \\
C1 & $0.0572(5)$ & $0.7649(4)$ & $0.19904(18)$ & $0.0302(5)$ \\
N2 & $0.1472(5)$ & $0.7573(4)$ & $0.09653(16)$ & $0.0377(6)$ \\
H9A & 0.0747 & 0.8643 & 0.0622 & $0.057^{*}$ \\
H9B & 0.0816 & 0.6259 & 0.0641 & $0.057^{*}$ \\
H9C & 0.3402 & 0.7770 & 0.1008 & $0.057^{*}$ \\
C3 & $-0.1968(5)$ & $0.9320(4)$ & $0.3237(2)$ & $0.0329(6)$ \\
C7 & $-0.3747(6)$ & $1.0962(5)$ & $0.3564(2)$ & $0.0391(6)$ \\
C6 & $0.1466(6)$ & $0.6149(5)$ & $0.2669(2)$ & $0.0374(6)$ \\
H6A & 0.2625 & 0.5081 & 0.2475 & $0.045^{*}$ \\
N1 & $-0.5144(6)$ & $1.2233(5)$ & $0.3856(2)$ & $0.0545(7)$ \\
C4 & $-0.1099(6)$ & $0.7815(5)$ & $0.3928(2)$ & $0.0395(6)$ \\
H4A & -0.1675 & 0.7865 & 0.4580 & $0.047^{*}$ \\
C2 & $-0.1148(5)$ & $0.9263(4)$ & $0.2256(2)$ & $0.0322(6)$ \\
H2A & -0.1732 & 1.0271 & 0.1794 & $0.039^{*}$ \\
C5 & $0.0624(7)$ & $0.6251(5)$ & $0.3633(2)$ & $0.0443(7)$
\end{tabular}


Atomic displacement parameters $\left(\AA^{2}\right)$

\begin{tabular}{lllllll}
\hline & $U^{11}$ & $U^{22}$ & $U^{33}$ & $U^{12}$ & $U^{13}$ & $U^{23}$ \\
\hline C11 & $0.0519(5)$ & $0.0373(4)$ & $0.0395(4)$ & $0.0155(3)$ & $0.0126(3)$ & $0.0062(3)$ \\
C1 & $0.0334(12)$ & $0.0304(13)$ & $0.0285(12)$ & $0.0073(10)$ & $0.0074(10)$ & $0.0027(9)$ \\
N2 & $0.0446(13)$ & $0.0398(13)$ & $0.0324(12)$ & $0.0139(10)$ & $0.0107(10)$ & $0.0040(9)$ \\
C3 & $0.0308(12)$ & $0.0338(14)$ & $0.0360(13)$ & $0.0102(10)$ & $0.0063(10)$ & $0.0020(10)$ \\
C7 & $0.0404(14)$ & $0.0421(16)$ & $0.0380(14)$ & $0.0140(12)$ & $0.0093(12)$ & $0.0045(11)$ \\
C6 & $0.0442(15)$ & $0.0333(14)$ & $0.0388(14)$ & $0.0165(11)$ & $0.0094(11)$ & $0.0065(11)$ \\
N1 & $0.0597(17)$ & $0.0551(17)$ & $0.0558(17)$ & $0.0274(14)$ & $0.0191(14)$ & $0.0036(13)$ \\
C4 & $0.0491(16)$ & $0.0429(16)$ & $0.0307(13)$ & $0.0136(12)$ & $0.0130(12)$ & $0.0063(11)$ \\
C2 & $0.0325(13)$ & $0.0316(13)$ & $0.0346(13)$ & $0.0099(10)$ & $0.0048(10)$ & $0.0062(10)$ \\
C5 & $0.0577(18)$ & $0.0420(17)$ & $0.0398(15)$ & $0.0216(13)$ & $0.0132(13)$ & $0.0154(12)$
\end{tabular}

Geometric parameters $\left(\AA,{ }^{o}\right)$

\begin{tabular}{|c|c|c|c|}
\hline $\mathrm{C} 1-\mathrm{C} 6$ & $1.380(4)$ & $\mathrm{C} 3-\mathrm{C} 7$ & $1.440(4)$ \\
\hline $\mathrm{C} 1-\mathrm{C} 2$ & $1.385(3)$ & $\mathrm{C} 7-\mathrm{N} 1$ & $1.139(4)$ \\
\hline $\mathrm{C} 1-\mathrm{N} 2$ & $1.460(3)$ & $\mathrm{C} 6-\mathrm{C} 5$ & $1.374(4)$ \\
\hline $\mathrm{N} 2-\mathrm{H} 9 \mathrm{~A}$ & 0.89 & C6-H6A & 0.93 \\
\hline $\mathrm{N} 2-\mathrm{H} 9 \mathrm{~B}$ & 0.89 & $\mathrm{C} 4-\mathrm{C} 5$ & $1.374(4)$ \\
\hline $\mathrm{N} 2-\mathrm{H} 9 \mathrm{C}$ & 0.89 & $\mathrm{C} 4-\mathrm{H} 4 \mathrm{~A}$ & 0.93 \\
\hline $\mathrm{C} 3-\mathrm{C} 4$ & $1.390(4)$ & $\mathrm{C} 2-\mathrm{H} 2 \mathrm{~A}$ & 0.93 \\
\hline $\mathrm{C} 3-\mathrm{C} 2$ & $1.391(4)$ & $\mathrm{C} 5-\mathrm{H} 5 \mathrm{~A}$ & 0.93 \\
\hline $\mathrm{C} 6-\mathrm{C} 1-\mathrm{C} 2$ & $121.9(2)$ & $\mathrm{C} 5-\mathrm{C} 6-\mathrm{C} 1$ & $119.2(2)$ \\
\hline $\mathrm{C} 6-\mathrm{C} 1-\mathrm{N} 2$ & $119.8(2)$ & $\mathrm{C} 5-\mathrm{C} 6-\mathrm{H} 6 \mathrm{~A}$ & 120.4 \\
\hline $\mathrm{C} 2-\mathrm{C} 1-\mathrm{N} 2$ & $118.3(2)$ & $\mathrm{C} 1-\mathrm{C} 6-\mathrm{H} 6 \mathrm{~A}$ & 120.4 \\
\hline $\mathrm{C} 1-\mathrm{N} 2-\mathrm{H} 9 \mathrm{~A}$ & 109.5 & $\mathrm{C} 5-\mathrm{C} 4-\mathrm{C} 3$ & $119.1(2)$ \\
\hline $\mathrm{C} 1-\mathrm{N} 2-\mathrm{H} 9 \mathrm{~B}$ & 109.5 & $\mathrm{C} 5-\mathrm{C} 4-\mathrm{H} 4 \mathrm{~A}$ & 120.4 \\
\hline $\mathrm{H} 9 \mathrm{~A}-\mathrm{N} 2-\mathrm{H} 9 \mathrm{~B}$ & 109.5 & $\mathrm{C} 3-\mathrm{C} 4-\mathrm{H} 4 \mathrm{~A}$ & 120.4 \\
\hline $\mathrm{C} 1-\mathrm{N} 2-\mathrm{H} 9 \mathrm{C}$ & 109.5 & $\mathrm{C} 1-\mathrm{C} 2-\mathrm{C} 3$ & $117.5(2)$ \\
\hline $\mathrm{H} 9 \mathrm{~A}-\mathrm{N} 2-\mathrm{H} 9 \mathrm{C}$ & 109.5 & $\mathrm{C} 1-\mathrm{C} 2-\mathrm{H} 2 \mathrm{~A}$ & 121.2 \\
\hline $\mathrm{H} 9 \mathrm{~B}-\mathrm{N} 2-\mathrm{H} 9 \mathrm{C}$ & 109.5 & $\mathrm{C} 3-\mathrm{C} 2-\mathrm{H} 2 \mathrm{~A}$ & 121.2 \\
\hline $\mathrm{C} 4-\mathrm{C} 3-\mathrm{C} 2$ & $121.3(2)$ & $\mathrm{C} 6-\mathrm{C} 5-\mathrm{C} 4$ & $121.0(2)$ \\
\hline $\mathrm{C} 4-\mathrm{C} 3-\mathrm{C} 7$ & $118.2(2)$ & $\mathrm{C} 6-\mathrm{C} 5-\mathrm{H} 5 \mathrm{~A}$ & 119.5 \\
\hline $\mathrm{C} 2-\mathrm{C} 3-\mathrm{C} 7$ & $120.4(2)$ & $\mathrm{C} 4-\mathrm{C} 5-\mathrm{H} 5 \mathrm{~A}$ & 119.5 \\
\hline $\mathrm{N} 1-\mathrm{C} 7-\mathrm{C} 3$ & $177.6(3)$ & & \\
\hline $\mathrm{C} 2-\mathrm{C} 1-\mathrm{C} 6-\mathrm{C} 5$ & $0.2(4)$ & $\mathrm{N} 2-\mathrm{C} 1-\mathrm{C} 2-\mathrm{C} 3$ & $-179.6(2)$ \\
\hline $\mathrm{N} 2-\mathrm{C} 1-\mathrm{C} 6-\mathrm{C} 5$ & $179.4(3)$ & $\mathrm{C} 4-\mathrm{C} 3-\mathrm{C} 2-\mathrm{C} 1$ & $0.0(4)$ \\
\hline $\mathrm{C} 2-\mathrm{C} 3-\mathrm{C} 4-\mathrm{C} 5$ & $0.5(4)$ & $\mathrm{C} 7-\mathrm{C} 3-\mathrm{C} 2-\mathrm{C} 1$ & $179.8(2)$ \\
\hline $\mathrm{C} 7-\mathrm{C} 3-\mathrm{C} 4-\mathrm{C} 5$ & $-179.3(3)$ & $\mathrm{C} 1-\mathrm{C} 6-\mathrm{C} 5-\mathrm{C} 4$ & $0.3(5)$ \\
\hline $\mathrm{C} 6-\mathrm{C} 1-\mathrm{C} 2-\mathrm{C} 3$ & $-0.3(4)$ & $\mathrm{C} 3-\mathrm{C} 4-\mathrm{C} 5-\mathrm{C} 6$ & $-0.6(5)$ \\
\hline
\end{tabular}




\section{supporting information}

Hydrogen-bond geometry $\left(\AA,{ }^{\circ}\right)$

\begin{tabular}{lllll}
\hline$D-\mathrm{H} \cdots A$ & $D-\mathrm{H}$ & $\mathrm{H} \cdots A$ & $D \cdots A$ & $D-\mathrm{H} \cdots A$ \\
\hline $\mathrm{N} 2-\mathrm{H} 9 A \cdots \mathrm{C} 11^{\mathrm{i}}$ & 0.89 & 2.61 & $3.111(3)$ & 116 \\
$\mathrm{~N} 2-\mathrm{H} 9 A \cdots \mathrm{C} 11^{\mathrm{ii}}$ & 0.89 & 2.65 & $3.178(3)$ & 119 \\
$\mathrm{~N} 2-\mathrm{H} 9 \boldsymbol{C}^{\mathrm{C}} \mathrm{Cl1}{ }^{\mathrm{iii}}$ & 0.89 & 2.73 & $3.278(3)$ & 121 \\
$\mathrm{~N} 2-\mathrm{H} 9 B \cdots \mathrm{Cl} 1$ & 0.89 & 2.76 & $3.338(3)$ & 124 \\
\hline
\end{tabular}

Symmetry codes: (i) $-x,-y+1,-z$; (ii) $x, y+1, z$; (iii) $-x+1,-y+1,-z$. 